\title{
Permanent Pacemaker Implantation Through Persistent Left Superior Vena Cava
}

\author{
Abdul Mueed ${ }^{1}$ and Muhammad Sarim Ansari ${ }^{2 *}$ \\ ${ }^{1}$ Department of Cardiac Electrophysiology, National Institute of Cardiovascular Diseases, Pakistan \\ ${ }^{2}$ Department of Cardiac Electrophysiology, National Institute of Cardiovascular Diseases, Pakistan
}

*Corresponding author: Muhammad Sarim Ansari, Department of Cardiac Electrophysiology, National Institute of Cardiovascular Diseases, Karachi, Pakistan.

Received Date: June 01, 2021

Published Date: July 15, 2021

\begin{abstract}
The left superior vena cava is a persistent embryo-genetic variant, occurring in 0.3 to $0.5 \%$ of individuals in which the venous blood of the upper half of the body is drained through a left vena cava into the coronary sinus and/or through the atrio-ventricular groove in the right atrium. We present a case of incidental finding of persistent left superior vena cava (PLSVC) in a 70 years female who was being implanted with a single chamber pacemaker for complete heart block.
\end{abstract}

Keywords: Permanent Pacemaker; Persistent Left Superior Vena Cava; Complete Heart Block

\section{Case Presentation}

70 year old female patient known to have hypertension for last 15 year compliant to medication came to emergency room with complains of shortness of breath and dizziness for last 15 days. Electrocardiogram (ECG) showed complete heart block for which she was placed on temporary intravenous pacing through right subcalvian venous approach. Work up was done for which no reversible cause was noted. Impression of senile deterioration of conducting tissue was made. Single chamber pacemaker implantation was planned. Lead was placed through the left subclavian vein. Access was performed via direct puncture; the guide-wire was advanced through left subclavian vein to persistent left superior vena cava and coronary sinus to right atrium. This was incidental finding which was confirmed during the procedure through venogram from left upper limb. Lead (Medtronic CapSureFix MRI active fixation $58 \mathrm{~cm}$ lead, Minneapolis, USA) was actively fixed in right ventricle apex with alpha loop configuration (Figure $1 \& 2$ ).

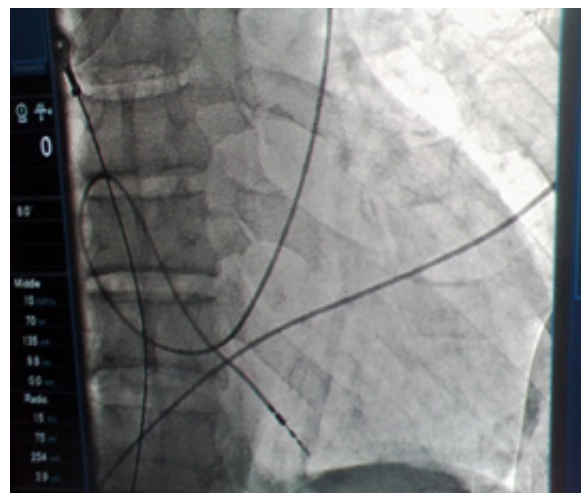

Figure 1: Cath Antero-posterior (AP) view. 


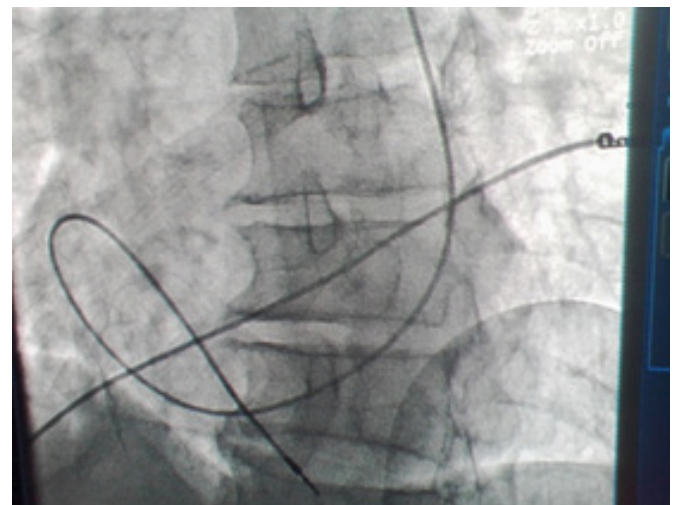

Figure 2: Cath Left Anterior Oblique (LAO) view.

The patient tolerated the procedure well and there were no complications. A chest X-ray obtained after procedure showed satisfactory lead positioning and no pneumothorax. At followup, she was noted to be in good health and her pacemaker was functioning normally.

\section{Discussion}

Persistent left superior vena cava (PLSVC) is a congenital vascular anomaly, occurring in 0.3 to $0.5 \%$ of individuals in the general population [1]. During embryogenesis, the sinus venosus consists of the right and left horns. Each receives blood from the common cardinal, vitelline and umbilical veins. During gestation, the left horn, after obliteration of the above veins, evolves into the coronary sinus and oblique vein of the left atrium, while the right becomes incorporated into the right atrium. The right common cardinal vein and the proximal part of the right anterior cardinal vein build the right superior vena cava. The left anterior cardinal vein changes into the internal jugular vein. The presence of the left anterior cardinal vein and obliteration of the left common cardinal vein leads to the formation of the left superior vena cava, which drains into the right atrium through the coronary sinus [2,3]. PLSVC is responsible for approximately $20 \%$ of the total venous blood return from the left arm, left half of the head and neck. In 80-90 $\%$ cases it drains into right atrium generally through CS resulting in CS dilation which can compress AV node or His bundle causing arrhythmias. PLSVC is associated with variety of congenital cardiac anomalies, such as anomalous connections of the pulmonary veins, aortic coarctation, tetralogy of Fallot, transposition of the great vessels as well as dextroversion [4-6]. Transvenous permanent pacemaker implantation in patients with PLSVC is challenging because of the complex anatomy. The coronary sinus (CS) may be dilated, which render pacing leads positioning from the left subclavian region difficult, especially the ventricular lead.

The use of active fixation leads with special curved stylet may help in overcoming this technical difficulty [7]. PLSVC is usually asymptomatic and is detected when cardiovascular imaging is performed for unrelated reasons. On chest X-ray, PLSVC can be seen as a widened shadow of the aorta with a visible venous halfmoon shadow from the left side of the aortic arch to the middle of the left clavicle. Basic diagnostic methods include transesophageal and transthoracic echocardiography. However, in our case report, PLSVC was diagnosed incidentally during pacemaker implantation which was confirmed through venogram during the procedure and as this finding was missed on pre- procedure transthoracic echocardiography. Right ventricular lead was successfully implanted with alpha loop configuration.

\section{Acknowledgement}

None.

\section{Conflict of Interest}

No conflict of interest.

\section{References}

1. Povoski SP, Khabiri H (2011) Persistent left superior vena cava: review of the literature, clinical implications, and relevance of alterations in thoracic central venous anatomy as pertaining to the general principles of central venous access device placement and venography in cancer patients. World J Surg Oncol 9: 173.

2. Pasquini L, Belmar C, Seale A, Gardiner HM (2006) Prenatal diagnosis of absent right and persistent left superior vena cava. Prenat Diagn 26(8): 700-702.

3. Miraldi F, di Gioiga CR, Proietti P, De Santis M, D Amati G, et al. (2002) Cardinal vein isomerism: an embryological hypothesis to explain a persistent left superior vena cava draining into the roof of the left atrium in the absence of coronary sinus and atrial septal defect. Cardiovasc Pathol 11(3): 149-152.

4. Zhong YL, Long X-M, Jiang L-Y, He B-F, Lin H, et al. (2015) Surgical treatment of dextroversion, isolated persistent left superior vena cava draining into the left atrium. J Card Surg 30(10): 767-770.

5. Kula S, Cevik A, Sanli C, Pektas A, Tunaoglu FS, et al. (2011) Persistent left superior vena cava: experience of a tertiary health-care center. Pediatr Int 53(6): 1066-1069.

6. Buirski G, Jordan SC, Joffe HS, Wilde P (1986) Superior vena caval abnormalities: their occurrence rate, associated cardiac abnormalities and angiographic classification in a paediatric population with congenital heart disease. Clin Radiol 37(2): 131-138.

7. Feldman A, Antonelli D, Turgeman Y (2013) Successful right ventricular lead placement using a right ventricular septal stylet in a patient with persistent left superior vena cava. EUROPACE 15: 354 